\title{
THE CLASSIFICATION OF FAINT Be STARS
}

\author{
DAVID A. ALLEN \\ Royal Greenwich Observatory, Anglo-Australian Observatory, Epping, New South Wales, Australia
}

(Paper read by J. P. Swings)

\begin{abstract}
Faint emission-line stars found on $\mathrm{H} \alpha$ surveys are usually classified as planetary nebulae or $\mathrm{Be}$ stars on rather arbitrary criteria. A comparison of spectra of such stars with their optical and infrared properties suggests that a next step in their classification can be made quite rapidly by photometry. In particular the more unusual specimens can be readily isolated.
\end{abstract}

Paul Merrill was the first person to engineer a systematic $\mathrm{H} \alpha$ survey of the northern sky as a means of identifying Be stars. That his technique was successful is beyond question; the three volumes of the MWC (Merrill and Burwell, 1933, 1943, 1949) testify to that.

But Merrill was bedevilled by one problem: how to distinguish his cherished $\mathrm{Be}$ stars from the chaff of emission-line objects that litter the sky. Nebulous objects compact $\mathrm{H}$ il regions and large planetary nebulae - he could hope to isolate simply by their extended nature; stellar objects - T Tauri stars, symbiotic stars, Me stars, compact planetary nebulae, Wolf-Rayet stars etc. - could be distinguished only spectroscopically. On the original objective-prism plates this was feasible, and was indeed undertaken, for the brightest sources, but it became increasingly difficult as the plate limit was neared. Merrill's solution was to publish a list of additional stars (the AS; Merrill and Burwell, 1950) about which he could say no more than that they had $\mathrm{H} \alpha$ emission. This is an admirable approach which has subsequently been shunned by the majority of $\mathrm{H} \alpha$ surveyors who seem to prefer risking errors of commission to making admissions which might be construed to imply inadequacy. Thus, rather than hiving off a 'don't know' group qua the AS, most recent $\mathrm{H} \alpha$ surveys have turned up only 'Be stars' or 'planetary nebulae'. The reasoning seems to be either: I see a continuum, ergo this is a Be star or: I see no continuum; this object has no continuum, ergo it is a planetary nebula. The outcome of this approach is to cram the Be star lists with T Tauri and even later-type stars, and the planetary nebula catalogues with the most bizarre assortment of objects, including even some $M$ stars with pure absorption spectra (Allen and Fosbury, 1975).

Even the impeccable MWC includes a number of stars Merrill chose to classify as Be but which could now better be described as forbidden-line stars. I refer to objects like MWC 17 (Swings and Struve, 1941), MWC 342 (Swings and Struve, 1943), MWC 645 and MWC 819 (Swings and Allen, 1973) in which low-excitation forbidden lines dominate the emission spectrum and there is a strong continuum offering little or no evidence of an underlying B-type star. The study of these enigmatic objects would be greatly benefited by their isolation from Be catalogues, but this was not always possible from the original Mount Wilson plates.

A problem therefore exists. The promulgators of objective prism surveys in the main seem unable or unwilling to classify their discoveries into more than two loosely defined categories. To do so spectroscopically requires an inordinate amount of 
telescope time and offers a low yield of the frontier-pushing astronomy we are supposed to be pursuing. How should we proceed?

A solution offered here is photometry which, by dint of its inherently wider bandpass, is considerably faster than spectroscopy and can be performed on a smaller telescope. Considering first the optical, filter combinations which have already proved successful and which could serve usefully in the classification of emission-line stars include the $\mathrm{H} \beta /[\mathrm{O} \mathrm{III}]$ and the $\mathrm{TiO} / \mathrm{CaH}$ sets. The first of these combinations was used by Webster (1966) to examine the stellar planetary nebulae of Henize's (1967) survey. On this basis she isolated 15 which had at best only very weak [O III] emission and which therefore merited at least a more thorough examination before they were accepted as genuine planetary nebulae. Two thirds of these have since been shown not to be planetary nebulae, and several of them would more properly be classed as Be stars. An $\mathrm{H} \beta /\left[\mathrm{O}_{\mathrm{III}}\right]$ photometric survey of all the objects classified as stellar or compact planetary nebulae would therefore aid in cleaning up this group. Late type stars - M, Me and symbiotic stars - can be identified by the $\mathrm{TiO} / \mathrm{CaH}$ photometry practiced by Jones (1973), or by similar filter combinations. Many emission-line stars are quite heavily reddened, so working in the red has obvious advantages. Since late-type stars are present in both samples, all faint emission-line stars found by objective prism surveys would need to be examined.

1-4 $\mu \mathrm{m}$ infrared photometry offers another, and perhaps a superior, means of separating the various emission-line objects (Allen and Swings, 1972). At the time of writing, most infrared observations of emission-line stars have been made with $\mathrm{PbS}$ cells; the advent of InSb, which is nearly an order of magnitude more sensitive, will considerably speed up this type of work. The standard Johnson filters at $1.25(\mathrm{~J})$, $1.65(H), 2.2(K)$ and $3.5 \mu \mathrm{m}(L)$ have been used. At these wavelengths three types of continuum can be identified, and these are listed in Table I.

TABLE I

The three varieties of infrared continua in emission-line stars

\begin{tabular}{|c|c|c|c|c|c|}
\hline \multirow[t]{2}{*}{ Type } & \multicolumn{4}{|c|}{$\begin{array}{l}\text { Typical colour indices } \\
\text { (magnitudes) }\end{array}$} & \multirow[t]{2}{*}{$\begin{array}{l}\text { Emission-line objects } \\
\text { represented }\end{array}$} \\
\hline & $J-H$ & $H-K$ & $K-L$ & $V-K$ & \\
\hline $\begin{array}{l}\text { i Blue continuum, } \\
\text { stellar or free-free }\end{array}$ & $\lesssim 0.3$ & $\$ 0.4$ & $\$ 0.6$ & $\sim 2$ & $\begin{array}{l}\text { Be stars; planetary } \\
\text { nebulae; most Wolf-Rayet } \\
\text { stars }\end{array}$ \\
\hline ii Late-type star & 1.0 & 0.4 & 0.4 & $3-15$ & $\begin{array}{l}\text { M, Me stars; most symbiotic } \\
\text { stars; VV cephei stars } \\
\text { except that } K-L \text { is usually } \\
\text { larger. Stars of type (i) } \\
\text { if } A_{V} \simeq 10 \text { mag. }\end{array}$ \\
\hline $\begin{array}{l}\text { iii Dust emission at } \\
\text { colour temperatures } \\
700-1500 \mathrm{~K}\end{array}$ & \multicolumn{4}{|c|}{$\begin{array}{l}\text { Colours depend on dust } \\
\text { temperature } \\
\qquad>0.6>0.9\end{array}$} & $\begin{array}{l}\text { Some dense planetary } \\
\text { nebulae; some symbiotic } \\
\text { stars; T Tauri stars; } \\
\text { forbidden-line stars; } \\
\text { compact H II regions; } \\
\text { pre-main-sequence Ae } \\
\text { and Be stars }\end{array}$ \\
\hline
\end{tabular}


The technique so far adopted (Allen, 1974; Allen and Glass, 1974, 1975) was to integrate for 5 minutes at $2.2 \mu \mathrm{m}$ using a PbS detector on a 1 or $1.2-\mathrm{m}$ telescope. In this time the majority of the late-type and dust emission objects will have been detected. Other wavelengths may then be tackled to derive colours. A one-minute integration with an InSb cell would go deeper, and hence find the fainter late-type and dust emission stars. Five minutes integrating on a similar aperture telescope would reach the continuum of many of the faint $B e$ stars and planetary nebulae. Thus infrared photometry offers a particularly rapid diagnostic for faint emission-line stars and serves to isolate the more unusual and peculiar specimens for further study. The data in Table II indicates the numbers of misclassified objects isolated by the author

TABLE II

Proportion of type (ii) and type (iii) infrared continua amongst emissionline stars fainter than about 13th magnitude

\begin{tabular}{lccl}
\hline & $\begin{array}{l}\text { Total } \\
\text { sampled }\end{array}$ & \%(ii) & $\%$ (iii) \\
\hline Classed as Be & 85 & 32 & 36 \\
Classed as planetary nebulae & 376 & 21 & 10 \\
\hline
\end{tabular}

in his various infrared surveys of Be stars and compact planetary nebulae, and illustrates the magnitude of the misclassification problem. The use of InSb detectors on the sources undetected with $\mathrm{PbS}$ cells would probably lead to an increase in the percentages of misclassified sources, especially amongst planetary nebulae.

\section{References}

Allen, D. A.: 1974, Monthly Notices Roy. Astron. Soc. 168, 1.

Allen, D. A. and Fosbury, R. A. E.: 1975, Observatory 95, 15.

Allen, D. A. and Glass, I. S.: 1974, Monthly Notices Roy. Astron. Soc. 167, 331.

Allen, D. A. and Glass, I. S.: 1975, Monthly Notices Roy. Astron. Soc. 170, 579.

Allen, D. A. and Swings, J. P.: 1972, Astrophys. Letters 10, 83.

Henize, K. G.: 1967, Astrophys. J. Suppl. 14, 125.

Jones, D. H. P.: 1973, Monthly Notices Roy. Astron. Soc. 161, 19P.

Merrill, P. W. and Burwell, C. G.: 1933, Astrophys. J. 78, 87.

Merrill, P. W. and Burwell, C. G.: 1943, Astrophys. J. 98, 153.

Merrill, P. W. and Burwell, C. G.: 1949, Astrophys. J. 110, 387.

Merrill, P. W. and Burwell, C. G.: 1950, Astrophys. J. 112, 72.

Swings, J. P. and Allen, D. A.: 1973, Astrophys. Letters 14, 65.

Swings, P. and Struve, O.: 1941, Astrophys. J. 93, 349.

Swings, P. and Struve, O.: 1943, Astrophys. J. 97, 194.

Webster, B. L.: 1966, Publ. Astron. Soc. Pacific 78, 136. 